\title{
Immunological evaluation of two novel engineered Plasmodium vivax circumsporozoite proteins formulated with different human-compatible vaccine adjuvants in C57BL/6 mice
}

\author{
Samaneh H. Shabani ${ }^{1,2}$. Sedigheh Zakeri ${ }^{1} \cdot$ Yousef Mortazavi $^{2} \cdot$ Akram A. Mehrizi $^{1}$
}

Received: 5 October 2018 / Accepted: 1 April 2019 / Published online: 25 April 2019

(c) Springer-Verlag GmbH Germany, part of Springer Nature 2019

\begin{abstract}
A vaccine targeting Plasmodium vivax signifies an additional necessary tool when considering the malaria elimination/ eradication goal. In this study, in vivo immunological evaluation of two novel engineered proteins of $P$. vivax circumsporozoite (PvCS127 and PvCS712) with two different arrangements of the repeat sequences of VK210 and VK247 was assessed. The immunological properties of the Escherichia coli-expressed chimeric proteins were evaluated by the immunization of C57BL/6 mice administered in NLX, CpG-ODNs, and QS21, alone or in combination as adjuvants. A significant increase in anti-rPvCS127 and -rPvCS712 IgG antibodies was observed in all the vaccine groups after the first boost, and the predominant isotypes were high-avidity cytophilic antibodies, $\mathrm{IgG} 2 \mathrm{~b}$, and $\mathrm{IgG} 2 \mathrm{c}$. The highest ratio of $\mathrm{IgG} 2 \mathrm{~b} / \mathrm{IgG1}(2.74)$ and $\mathrm{IgG} 2 \mathrm{c} / \mathrm{IgG} 1$ (2.1) levels was detected in mouse groups immunized with rPvCS712 + NLX-CpG-QS21. The lowest level of IFN- $\gamma$ (mean: 441 and $588 \mathrm{pg} / \mathrm{mL}$, respectively) was produced by the mouse group, which received both antigens without any adjuvant, while significant levels of IFN- $\gamma$ were detected in the mouse groups immunized with rPvCS127- or rPvCS712NLX-CpG-QS21 formulation (mean: 1200 and $3092 \mathrm{pg} / \mathrm{mL}$, respectively). The current results indicated that in C57BL/6 mice, both recombinant antigens were efficient immunogens and could induce humoral and cellular immune responses and their combination with three Th1 potent adjuvants had an impact on the magnitude and the quality of humoral responses (specific antibody subclasses, titer, and high avidity). Although the overall response was marginally higher for $\operatorname{rPvCS712}$ than $\mathrm{rPvCS} 127$, all immunized mice induced some immune responses against both proteins, and the present findings indicate that $\mathrm{rPvCS} 127$ and $\mathrm{rPvCS} 712$ meet the criteria to be potentially useful vaccine candidates against $P$. vivax malaria.
\end{abstract}

Keywords Plasmodium vivax $\cdot$ Vaccine $\cdot$ Circumsporozoite protein $\cdot \operatorname{rPvCS127} \cdot \operatorname{rPvCS} 712 \cdot$ Adjuvant

Edited by: Christian Bogdan.

Electronic supplementary material The online version of this article (https://doi.org/10.1007/s00430-019-00606-9) contains supplementary material, which is available to authorized users.

Sedigheh Zakeri

zakeris@yahoo.com; zakeris@pasteur.ac.ir

1 Malaria and Vector Research Group (MVRG), Biotechnology Research Center (BRC), Pasteur Institute of Iran, P.O. BOX 1316943551, Tehran, Iran

2 Department of Medical Biotechnology and Nanotechnology, Faculty of Medicine, Zanjan University of Medical Sciences (ZUMS), Zanjan, Iran

\section{Introduction}

Malaria still claims over half a million cases annually and has a significant negative impact on global healthcare as well as on the economy of malaria-endemic countries [1]. Although Plasmodium falciparum is the most virulent Plasmodium species, Plasmodium vivax (as the second most prevalent malaria species) spread over a wider geographical area outside Africa [2]. Malaria eradication research agenda (MalERA) has placed vivax vaccine development in its top list of priorities for malaria elimination and eradication strategies [3] due to the increase in $P$. vivax drug resistance isolates, the relapsing behavior, the presence of co-infections with other Plasmodium spp., and reports of severe and lethal cases [4-6]. Such a vaccine should be able to prevent infection and transmission [7]. Thus, the best target vaccine candidate antigen could be from the earliest stage of the 
malaria parasite in the human host since it has the potential to achieve both goals.

At the earliest stage of Plasmodium's life cycle, the infected Anopheles mosquito inoculates sporozoites into the skin during its blood meal, and within an hour, they reach the liver through the bloodstream where they invade hepatocyte cells [8]. The sporozoites are accessible to antibodies while they are in the skin and also in the bloodstream. Therefore, a pre-erythrocytic vaccine would prevent not only the entry of sporozoites into hepatocytes but also the development of hypnozoite form, hence inhibiting the infection and transmission of the parasite [9]. Recently, much effort has been focused on one of the sporozoite surface antigens, namely circumsporozoite protein (CSP). A small panel of broadly neutralizing antibodies isolated from Plasmodiuminfected individuals has helped identify the regions of CSP to be targeted by a malaria subunit vaccine. These regions include highly immunogenic central repetitive domains containing short blocks of nona tandem amino acid repeats. These repeat sequences consist of three different variants (VK210, VK247, and P. vivax-like) of which VK210 and VK247 have been reported from global vivax-endemic regions [10-12]. The central domain is flanked by two non-repetitive $\mathrm{N}$ - and $\mathrm{C}$-terminal regions containing small stretches of highly conserved sequences that are composed of B- and T cell epitopes [13-15]. Neutralizing antibodies against repeat regions [16, 17] and non-repeat regions [18] have been reported in the serum of malaria-exposed individuals. Therefore, it is supposed that a $P$. vivax CSP (PvCSP)specific neutralizing antibody is essential for immunity to $P$. vivax liver stages against sporozoite. However, eliciting broadly neutralizing antibodies is not an easy subject due to high allelic variations within PvCSP; therefore, using a single allele of CSP in PvCSP-based vaccine is likely ineffective and may induce incomplete and/or strain-specific immunity.

The development of a whole $P$. vivax vaccine has been postulated to be difficult, and one of the main reasons for the difficulty in its development is the lack of continuous in vitro culture of $P$. vivax parasite. It seems, at this time, that the only solution for the development of a vivax malaria vaccine could be subunit vaccine. In this regard, the immune responses elicited by recombinant vaccine antigens are short-lived with a limited capacity; therefore, additional appropriate immunostimulants, such as an efficient adjuvant, are highly important to induce a protective and longlived immune response [15, 19-22]. Selection of a proper adjuvant may be based on different parameters, including potential use in human, nature of the vaccine antigen, and the type of required immune response. It should be noted that in malaria, the type of immune response required for protection against this disease is the Th1 type, which has been well documented before [23-26]. Also, a study by
Kester et al. [27] has reported that there is an association between Th1 cytokine-expressing CD4+ T-cells and protection against malaria infection in the human challenge model that reinforces the requirement of Th1 type for the development of efficient malaria vaccines. In this concern, there are numerous experimental adjuvants under development that aim at inducing strong neutralizing antibodies and T-cell responses; however, due to the high level of toxicity and adverse side effects, a vast majority of adjuvants could not be used in human vaccine trials $[22,28]$. Thus, in malaria subunit vaccine development, the selection of adjuvant is critical to induce proper and protective immune responses as well as to increase Th1 type antibodies (IgG1 and IgG3 in human and $\mathrm{IgG} 2 \mathrm{a} / \mathrm{c}$ and $\operatorname{IgG} 2 \mathrm{~b}$ in mice) with greater avidity and Th1 cytokine [interferon gamma (IFN- $\gamma)$ ] production against the malaria parasite [29-31].

One of the adjuvants that could potentially be used in human and shift the immune response to Th1 response [32-38] is naloxone (NLX), which has been approved by the FDA as a prescription drug that rapidly and safely reverses opioid-induced respiratory depression (http://www.acces sdata.fda.gov/scripts/cder/drug-satfda/index.cfm). It appears that NLX has substantial immune-modulating effects on the immune system, which is mediated directly through opioid receptors on immune cells and/or indirectly through the central nervous system and the hypothalamic-pituitary-adrenal axis [39]. Opioid receptors have been detected on both $\mathrm{T}$ and B lymphocytes, dendritic cells (DCs), and other immune cell [39]. Another potent Th1 type adjuvant is CpG-ODNs (oligodeoxynucleotides) comprising short synthetic DNA molecules containing unmethylated $\mathrm{CpG}$ motifs and cytosine phosphoguanosine dinucleotides that are common in bacteria and virus [40]. This adjuvant stimulates innate immune responses and prompts the production of Th1 type cytokines such as IFN- $\gamma$ via interaction with antigen-presenting cells, endosomally through TLR9 such as DCs, macrophages, and B lymphocytes [41-44]. It has a high potential to induce both innate and specific humoral and Th1 cell-mediated responses [45]. Many studies have also shown that QS21, a saponin natural product from the Quillaja saponaria tree [46] with low toxicity, is one of the most promising vaccine adjuvants that is currently under investigation $[46,47]$. This adjuvant stimulates high antigen-specific antibody responses and CD8+ T-cell response in mouse [48-54]. Further studies have demonstrated that QS21 stimulates the trigger of cytotoxic T lymphocytes (CTLs), Th1 cytokines, and the IgG2a antibody isotype against protein antigens [50, 52, 55-57] via interaction with the cell membrane of antigen-presenting cells $[19,58]$.

In an attempt to develop an efficient vivax malaria subunit vaccine based on CSP [59-61], we have previously developed two recombinant chimeric antigens, on the basis of the two most widespread variant forms (VK210 and VK247), 
namely after rPvCS127 and rPvCS712 [62]. Both recombinant molecules encode a full-length molecule encompassing the $\mathrm{N}$ - and $\mathrm{C}$-terminal regions flanking a chimeric repeat region representing VK210 $(n=12)$ and VK247 $(n=7)$. In the current investigation, in continuation of our previous work [62] following the expression and purification of the two chimeric recombinant proteins in Escherichia coli ( $E$. coli), the potential usefulness of these proteins for the development of a protective vivax malaria vaccine was evaluated. The assessment was achieved through the immunization of C57BL/6 mice with chimeric antigens $\mathrm{rPvCS} 127$ and $\mathrm{rPvCS} 712$ delivered in three putative human-use compatible adjuvants: NLX, CpG-ODNs, and QS21 alone or in combination (NLX-CpG-QS21). Also, humoral immune responses (IgG, IgG1, IgG2b, IgG2c, and IgG3 responses) and their avidity, as well as the IFN- $\gamma$, IL-4, and IL-10 cytokines responses were determined in immunized mouse sera. Moreover, both induced humoral and cellular immunity responses were compared between immunized mouse groups that received $\mathrm{rPvCS} 127$ or $\mathrm{rPvCS} 712$ alone (nonadjuvanted vaccine groups) or with various human-use compatible potent adjuvants (adjuvanted vaccine groups), as well as with complete Freund's (CFA), as a reference adjuvant.

\section{Materials and methods}

\section{Expression, purification, and confirmation of engineered PvCS127 and PvCS712 in E. coli host}

Both rPvCS127 and rPvCS712 (GenBank accession nos. KY548403 and KY548404, respectively) were successfully cloned, expressed and purified as described earlier [62]. In brief, chimeras were designed, codon optimized and synthesized to express in E. coli expression vectors as a fusion protein with a C-terminal hexa-His tag. The protein expression was induced by isopropyl-beta-D-thiogalactopyranoside (IPTG, Thermo Scientific, Waltham, Massachusetts, USA) with continuous shaking $(180 \mathrm{rpm})$ at $37^{\circ} \mathrm{C}$ for $4 \mathrm{~h}$. The optimum conditions for $\mathrm{rPvCS} 127$ and $\mathrm{rPvCS} 712$ expression were in TB and $2 \times \mathrm{LB}$ media, respectively, where 0.5 and $0.2 \mathrm{mM}$ IPTG were added to the culture until $\mathrm{OD}_{600 \mathrm{~nm}}$ reached $\sim 1$. Both $\mathrm{rPvCS} 127$ and $\mathrm{rPvCS} 712$ were expressed in $E$. coli in adequate amounts for immunization $(\sim 5 \mathrm{mg})$, and after purification, the proteins were analyzed under reducing [with $1 \%$ sodium dodecyl sulfate (SDS) and $2 \%$ $\beta$-mercaptoethanol (2ME)] conditions by $12 \%$ SDS-polyacrylamide gel electrophoresis (SDS-PAGE). The results showed a single band with a molecular mass of $\sim 50 \mathrm{kDa}$ [62]. Also, Western blot analysis using anti-His antibody, peroxidase-linked anti-CSP-specific monoclonal antibodies (anti-Pv-210-CDC, P. vivax allelic form VK210 and anti-Pv-247-CDC, P. vivax allelic form VK247), and $P$. vivax-infected human sera recognized the $\mathrm{rPvCS} 127$ and rPvCS712 [62]. The level of bacterial endotoxin was determined using the LAL chromogenic kit (Lonza, Walkersville, MD, USA) in the Quality Control Unit of the Recombinant Protein Production Complex of Pasteur Institute of Iran (Karaj, Iran) and the endotoxin concentration of each of the purified recombinant proteins was less than $0.1 \mathrm{EU} / \mathrm{mL}$. Therefore, the injection of $200 \mu \mathrm{L}$ of purified $\mathrm{rPvCS} 127$ or $\mathrm{rPvCS} 712$ /mouse had an acceptable amount (0.02 EU) of endotoxin into each mouse.

\section{Mice immunization with rPvCS127and rPvCS712}

Six- to eight-week-old inbred female C57BL/6 mice were obtained from the Laboratory of Animal Science Department of the Pasteur Institute of Iran. The mice were housed under specific pathogen-free conditions in the animal care facility and allowed to adapt to housing conditions 1 week prior to the experiment. The experimental protocols were approved by the Committee of Animal Ethics of the Pasteur Institute of Iran and performed accordingly. All mice were randomly divided into 18 groups ( $n=15$ /group) and were immunized by subcutaneous injection of purified rPvCS127 or $\mathrm{rPvCS} 712(10 \mu \mathrm{g}$ at prime and $5 \mu \mathrm{g}$ at boost) alone (nonadjuvanted vaccine groups) or in combination with NLX, CpG-ODNs 1826 (TCCATGACGTTCCTGACGTT), and QS21 as adjuvants (adjuvanted vaccine groups) at the base of the tail, three times at 14-day intervals (Table 1 and Supplementary Fig. S1). In the negative control groups, the mice were similarly immunized with sterile PBS $(0.5 \times \mathrm{PBS}$, $\mathrm{pH}$ 7.4), NLX (5 mg/kg mouse; Sigma-Aldrich Co., St. Louis, MO, USA), CpG-ODN 1826 (2 mg/mL; InvivoGen, San Diego, CA, USA), QS21 (1 mg/mL; Alpha Diagnostic Intl. Inc, San Antonio, Texas, USA) and also with the combination of NLX (5 mg/kg mouse)-CpG (5 $\mu \mathrm{g} /$ mouse)-QS21 (5 $\mu \mathrm{g} /$ mouse) (NLX-CpG-QS21; Table 1). The mice were then immunized with $\mathrm{PPvCS} 127$ or $\mathrm{rPvCS} 712$ emulsified in CFA as the reference adjuvant (the first dose) or IFA (subsequent doses), both from Sigma-Aldrich Co., with 1:1 volume/volume prior to the injection. For the evaluation of the humoral responses to $\mathrm{rPvCS} 127$ and $\mathrm{rPvCS} 712$, the serum samples were collected from the tail vein before immunization (as pre-immune sera) as well as 10 days after each immunization (day 10, after the first injection; day 24, after the first boost; day 38, after the second boost; Table 1 and Supplementary Fig. S1). All the sera were stored at $-20{ }^{\circ} \mathrm{C}$ until use.

\section{Measurement of antibody responses to $\mathrm{rPvCS} 127$ and rPvCS712}

Immunized mouse sera were evaluated for anti-rPvCS127or -rPvCS712-specific antibodies by ELISA as described 
Table 1 Mice immunization strategy

\begin{tabular}{|c|c|c|c|c|c|c|c|c|}
\hline \multicolumn{2}{|c|}{ Immunized mouse groups } & \multicolumn{7}{|c|}{ Antigen/adjuvant(s) } \\
\hline & & \multicolumn{3}{|c|}{$\operatorname{rPvCS} 127$ or $\operatorname{rPvCS} 712(\mu \mathrm{g})$} & \multirow{2}{*}{$\begin{array}{l}\text { CFA/IFA } \\
(\mu \mathrm{L})\end{array}$} & \multirow{2}{*}{$\begin{array}{l}\text { CpG ODN } \\
(\mu \mathrm{g} / \text { mouse })\end{array}$} & \multirow{2}{*}{$\begin{array}{l}\text { QS21 } \\
\text { ( } \mu \mathrm{g} / \text { mouse) }\end{array}$} & \multirow{2}{*}{$\begin{array}{l}\text { NLX } \\
\text { (mg/kg mouse) }\end{array}$} \\
\hline & & 1st I* (day 0) & 2nd I (day14) & 3rd I (day 28) & & & & \\
\hline 1 & rPvCS127(non-adjuvanted) & 10 & 5 & 5 & - & - & - & - \\
\hline 2 & rPvCS127 + CFA/IFA & 10 & 5 & 5 & 100 & - & - & - \\
\hline 3 & rPvCS127 + NLX-CpG-QS21 & 10 & 5 & 5 & - & 5 & 5 & 5 \\
\hline 4 & $\mathrm{rPvCS} 127+\mathrm{CpG}$ & 10 & 5 & 5 & - & 5 & - & - \\
\hline 5 & $\operatorname{rPvCS} 127+\mathrm{QS} 21$ & 10 & 5 & 5 & - & - & 5 & - \\
\hline 6 & rPvCS127+NLX & 10 & 5 & 5 & - & - & - & 5 \\
\hline 7 & rPvCS712 (non-adjuvanted) & 10 & 5 & 5 & - & - & - & - \\
\hline 8 & $\mathrm{rPvCS} 712+\mathrm{CFA} / \mathrm{IFA}$ & 10 & 5 & 5 & 100 & - & - & - \\
\hline 9 & rPvCS712 + NLX-CpG-QS21 & 10 & 5 & 5 & - & 5 & 5 & 5 \\
\hline 10 & $\mathrm{rPvCS} 712+\mathrm{CpG}$ & 10 & 5 & 5 & - & 5 & - & - \\
\hline 11 & $\mathrm{rPvCS} 712+\mathrm{QS} 21$ & 10 & 5 & 5 & - & - & 5 & - \\
\hline 12 & $\operatorname{rPvCS712+NLX}$ & 10 & 5 & 5 & - & - & - & 5 \\
\hline & Control groups & & & & & & & \\
\hline 13 & $\mathrm{CpG}$ & - & - & - & - & 5 & - & - \\
\hline 14 & QS21 & - & - & - & - & - & 5 & - \\
\hline 15 & NLX & - & - & - & - & - & - & 5 \\
\hline 1 & NLX-CpG-QS216 & - & - & - & - & 5 & 5 & 5 \\
\hline 17 & CFA/IFA & - & - & - & 100 & - & - & - \\
\hline 18 & PBS $0.5 \times$ & - & - & - & - & - & - & - \\
\hline
\end{tabular}

Mice received $200 \mu \mathrm{L}$ rPvCS127 or $\mathrm{rPvCS} 712(10 \mu \mathrm{g}$ at prime and $5 \mu \mathrm{g}$ at boost) alone (non-adjuvanted vaccine groups) and in the presence of CFA/IFA, CpG, OS21, and NLX adjuvants (adjuvanted vaccine groups) subcutaneously at the base of tail. Control mice received PBS, CFA/IFA (as the reference adjuvant), CpG, QS21, NLX, and NLX-CpG-QS21 combination. Serum samples were collected from the tail vein 10 days after each immunization (days 10, 24, and 38 after the first immunization)

$C F A$ complete Freund's adjuvant, IFA incomplete Freund's adjuvant, $N L X$ naloxone, $C p G O D N$ synthetic oligodeoxynucleotides (ODNs) containing unmethylated $C p G$ motifs, $Q S 21$ synthetic variant of the saponin natural product, I* injection

before [62] with some modifications. In brief, $\mathrm{rPvCS} 127$ or $\mathrm{rPvCS} 712$ protein $(50 \mathrm{ng} /$ well) was diluted in a coating buffer $\left(0.06 \mathrm{M}\right.$ of $\left.\mathrm{Na}_{2} \mathrm{CO}_{3} / \mathrm{NaHCO}_{3}, \mathrm{pH} 9.6\right)$, coated in MaxiSorp flat-bottom 96-well ELISA plates (Jet Biofil, Guangzhou, China) and kept at $4{ }^{\circ} \mathrm{C}$ overnight. After incubation, the plates were washed three times with PBS-T and were blocked with $1 \times$ PBS containing $1 \%$ BSA (Roche, Basel, Switzerland) at room temperature (RT) for $1 \mathrm{~h}$. After washing, the serum samples were incubated in duplicate wells with 1:200 diluted sera in PBS-T in 0.5\% BSA for $90 \mathrm{~min}$. The plates were washed and incubated with 100 $\mu \mathrm{L}$ of 1:25,000 dilutions of goat anti-mouse IgG antibodies conjugated with HRP (Sigma-Aldrich Co.) at RT for $60 \mathrm{~min}$. Bound anti-CSP IgG was visualized after adding o-phenylenediamine $\mathrm{H}_{2} \mathrm{O}_{2}$ (OPD, [Sigma-Aldrich Co.]) as the substrate. After 10-20 min, the reaction was stopped with $2 \mathrm{~N} \mathrm{H}_{2} \mathrm{SO}_{4}$, and $\mathrm{OD}_{490 \mathrm{~nm}}$ was read using an ELISA microplate reader (BioTek, Winooski, VT, USA). The ELISA cutoffs were obtained from the average of the preimmune mouse sera ( $n=20$, normal mouse sera) plus three standard deviations (SD). Moreover, for the measurement of the specific IgG subclasses response to $\mathrm{rPvCSP}$, the test was performed as described above, except for the secondary antibodies that were specific to mouse IgG1, IgG2b, IgG3 (Sigma-Aldrich Co.), and IgG2c (Abcam, Cambridge, U.K.) antibodies diluted 1:1000 and incubated at RT for $1 \mathrm{~h}$. After the washing step, the plates were incubated with 1:10,000 dilution of anti-goat IgG HRP (Sigma-Aldrich Co.) for the detection of $\operatorname{IgG}$ subclasses and then developed by the enzyme-specific substrate, as mentioned above.

\section{Anti-rPvCSP antibody avidity}

The avidity of anti-rPvCS127 and -rPvCS712 total IgG and its subclasses (IgG2b and $\operatorname{IgG} 2 \mathrm{c}$ ) was estimated using ELISA, as described by Hedman et al. [63] with minor modifications. In brief, MaxiSorp plates (Jet Biofil, Guangzhou, China) were coated with rPvCS127 or rPvCS712 protein (two plates for each, $50 \mathrm{ng} /$ well) and then blocked with $1 \%$ BSA for $60 \mathrm{~min}$. The plates were washed and incubated 
with mouse sera (1:200) at RT for 90 min. During washing step, one of the plates was washed three times with PBS-T, and the other plate was washed three times with dissociation buffer containing PBS-T-urea ( $8 \mathrm{M})$ with vigorous shaking. Next, both plates were washed once with an additional wash with PBS-T buffer. Finally, incubation with secondary antibody, washing steps, and development of enzyme reaction were performed as mentioned above for ELISA. The avidity index (AI) was calculated by the ratio of OD value of ureatreated samples to that of non-treated samples multiplied by 100 . AI values $\leq 30 \%$, between $30 \%$ and $50 \%$, and $>50 \%$ were considered as low-, intermediate-, and high-avidity anti-rPvCSP-specific antibodies, respectively.

\section{Measurement of lymphocyte proliferation by thiazolyl blue (MTT) assay}

The mouse lymphocyte proliferation was performed by 3-(4,5-Dimethylthiazol-2-yl)-2,5-diphenyltetrazolium bromide (MTT) dye assay. In detail, three mice from each vaccine and control groups were scarified by cervical dislocation under sterile conditions on day 42 after the first immunization. Then, the spleens were removed, and single-cell suspensions were prepared in RPMI 1640 medium (Gibco, Invitrogen, Scotland, UK). Red blood cells were osmotically eliminated with the ammonium chloride potassium lysis buffer ( $\mathrm{pH} 7.2$ ), and the cells were then washed twice and re-suspended in RPMI 1640 medium containing $5 \%$ fetal calf serum, $2.3 \times 10^{-2} \mathrm{mM}$ of $2-\mathrm{ME}, 100 \mathrm{U}-100 \mu \mathrm{g} /$ $\mathrm{mL}$ of penicillin-streptomycin, and $10 \mathrm{mM}$ of HEPES (Sigma-Aldrich Co.). The cell viability was confirmed by Trypan blue dye exclusion. In the next step, the splenocytes $\left(2.5 \times 10^{5} \mathrm{cell} /\right.$ well $)$ were cultured in a flat-bottom, 96 -well tissue culture plate (Orange Scientific, EU, Belgium) in triplicates in the presence of $\mathrm{rPvCS} 127$ or $\mathrm{rPvCS} 712$ (10 $\mu \mathrm{g} / \mathrm{mL}$ ), concanavalin A (ConA, $5 \mu \mathrm{g} / \mathrm{mL}$, as the positive control), and medium alone (as the negative control). Also, the cultivation of splenocytes from the non-immunized mice in the presence of $\mathrm{rPvCS} 127$ or $\mathrm{rPvCS} 712(10 \mu \mathrm{g} / \mathrm{mL})$ was used as a negative control. All plates were incubated in a humidified atmosphere in a $5 \% \mathrm{CO}_{2}$ incubator at $37{ }^{\circ} \mathrm{C}$ for $48 \mathrm{~h}$. Subsequently, the supernatants were removed, and cell proliferation was measured by MTT assay. In this assay, 100 $\mu \mathrm{L}$ of MTT $(0.5 \mathrm{mg} / \mathrm{mL}$, Sigma-Aldrich Co. $)$ was added to each well, and the plates were further incubated at $37{ }^{\circ} \mathrm{C}$ for $4 \mathrm{~h}$. The supernatant was removed, and the formazon crystals were dissolved in $0.04 \mathrm{~N}$ of $\mathrm{HCl}$ in absolute isopropanol, and the OD was measured at $550 \mathrm{~nm}$.

\section{Cytokine ELISA}

Murine cytokine immunoassay kits (R\&D system, Minneapolis, USA) were used to analyze cytokine release profiles in the supernatants of stimulated splenocytes of mice immunized with rPvCS127 or rPvCS712 constructs. On day 42 after the first immunization, three mice from each vaccine and control groups were scarified, and the spleens were isolated. After that, a single-cell suspension of splenocytes was seeded in a complete RPMI 1640 medium, supplemented with $5 \%$ fetal calf serum, $2 \mathrm{mM}$ of glutamine, $2.3 \times 10^{-2}$ $\mathrm{mM}$ of $2-\mathrm{ME}, 10 \mathrm{mM}$ of HEPES, and $100 \mathrm{U}-100 \mu \mathrm{g} / \mathrm{mL}$ of penicillin-streptomycin. The cells were incubated in flat-bottomed, 96-well microtiter plates (Orange Scientific, EU, Belgium) at a density of $2.5 \times 10^{5}$ cells/well in the presence of $\mathrm{rPvCS} 127$ or $\mathrm{rPvCS} 712(10 \mu \mathrm{g} / \mathrm{mL}$ for each). The positive and negative controls were ConA $(5 \mu \mathrm{g} / \mathrm{mL})$ and medium alone, respectively. All the plates were incubated in the presence of $5 \% \mathrm{CO}_{2}$ in a humidified atmosphere at $37^{\circ} \mathrm{C}$. Based on optimization-stimulation, spleen cells with various antigen concentrations and varying periods of time (ranging 24-144 h) were stimulated, and culture supernatants were collected at optimized time after 24 and $48 \mathrm{~h}$ for IL-4, $72 \mathrm{~h}$ for IL-10, and $120 \mathrm{~h}$ for IFN- $\gamma$. The concentration of cytokines was calculated based on the standard curves performed in parallel with the known concentrations of recombinant mouse IL-4, IL-10, and IFN- $\gamma$ cytokines for each experiment. All tests were performed in duplicate, and the mean concentration \pm SD was recorded for each set of samples.

\section{Statistical analysis}

A database was created using IBM SPSS 21.0 for Windows (Armonk, NY: IBM Corp, USA). The differences in the level of antibody responses in different vaccine mouse groups were analyzed using one-way ANOVA, followed by Tukey's honestly significant difference (HSD) post hoc test. Differences between time points were tested with paired sample $t$ test. $P<0.05$ was considered statistically significant $(* P<0.05, * * P \leq 0.001$, and $* * * P<0.0001)$.

\section{Results}

\section{Immunological evaluation of rPvCS127 and rPvCS712 antigens}

\section{$\lg G$ antibody responses}

By comparing the antibody responses to $\mathrm{rPvCS} 127$ and rPvCS712 antigens between the prime (day 10) and two boosts (days 24 and 38 ) in all vaccine mouse groups (1-12; Table 1), we found a significant increase in the anti-rPvCS127 (Fig. 1a) and -rPvCS712 (Fig. 1b) IgG antibodies levels on day 24 relative to day 10 after the first immunization $(P<0.05$, paired sample $t$ test). However, 
Fig. 1 Measurement of total anti-rPvCS127 (a) and -rPvCS712 (b) IgG antibody levels in vaccine mouse sera at different time points after immunization by ELISA. AntirPvCS127 and -rPvCS712 total IgG antibody levels were compared between the sera collected from immunized mice on days 10, 24 (10 days after the first boost), and 38 (10 days after the second boost) after the first immunization. The serum samples were incubated in duplicate wells with 1:200 diluted sera for $90 \mathrm{~min}$. No significant difference was observed in total IgG antibody levels between the first and the second boosts in adjuvanted vaccine groups (paired sample $t$-test, $P>0.05$ ). The highest levels of antirPvCS127 or -rPvCS712 IgG antibody were identified in the groups that received $\mathrm{rPvCS} 127$ or rPvCS712 with NLX-CpGQS21 combination, respectively $(P<0.0001$, one-way ANOVA $)$. The bars and error bars show the mean $\mathrm{OD}_{490 \mathrm{~nm}}$ and standard deviation (SD) in each mouse group $(n=15)$, respectively. The ELISA cutoffs were calculated as the mean $\mathrm{OD}_{490 \mathrm{~nm}}$ of pre-immune mouse sera (as negative controls, $n=20$ ) plus 3 $\mathrm{SD}$. The cutoffs for total IgG on days 10,24 , and 38 were almost $0.162(\mathrm{rPvCS} 127)$ and 0.175 (rPvCS712). $* P<0.05$
(A) Anti-rPvCS127 Total IgG Antibody

may 10

day 24

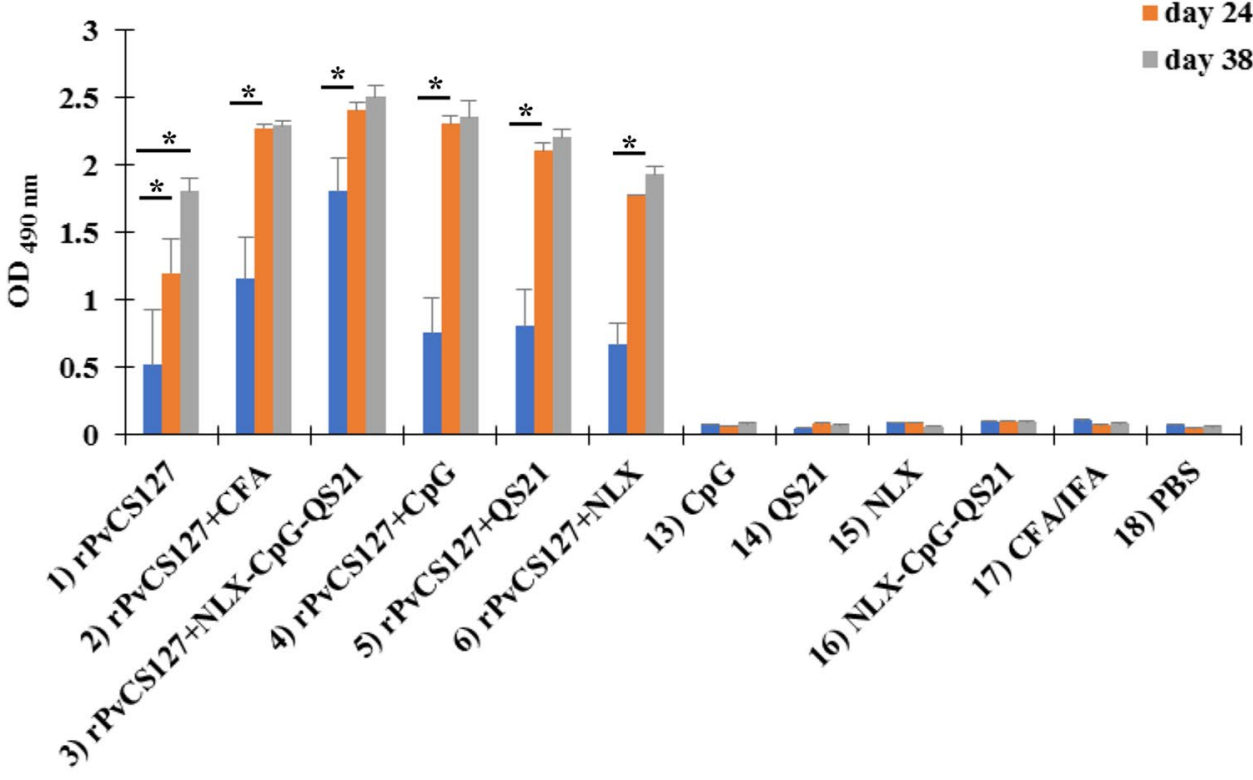

(B) Anti-rPvCS712 Total IgG Antibody

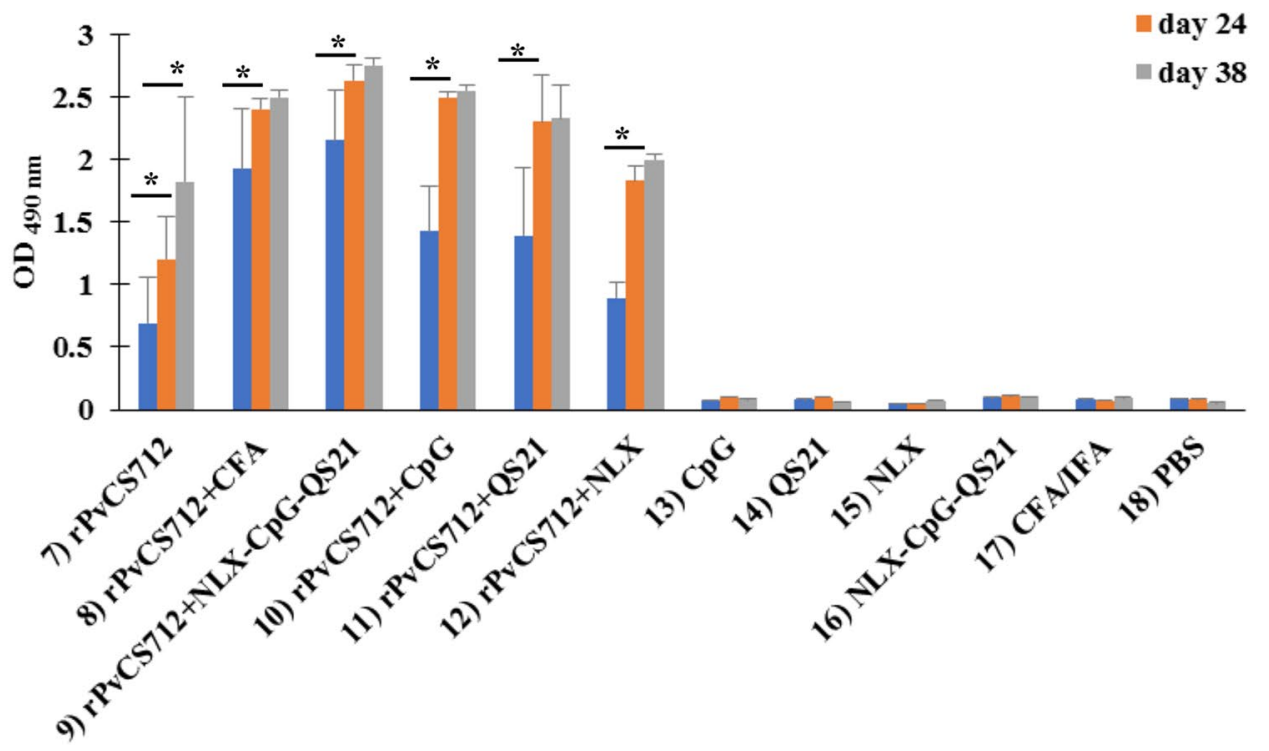

comparable anti-rPvCS127 or -rPvCS712 IgG antibodies were observed among the adjuvanted vaccine groups $(\mathrm{rPvCS} 127: 2-6$ and $\mathrm{rPvCS} 127: 8-12)$ on days 24 and 38 after the first immunization $(P>0.05$, paired sample $t$-test, Fig. 1a, b). Both the vaccine groups receiving antigen alone (1 and 7) induced anti-rPvCS127 and -rPvCS712 IgG antibodies in the immunized mice with no statistically significant difference $(P>0.05$, Tukey's HSD post hoc test, Fig. 2a). However, comparison of the nonadjuvanted ( 1 and 7$)$ with the adjuvanted (2-6 and 8-12) vaccine groups displayed a statistically significant increase in specific anti-PvCSP IgG antibodies levels in the adjuvanted vaccine groups $(P<0.0001$, one-way ANOVA; Fig. 2a). When comparing the antibody responses to rPvCS127 and PvCS712 antigens in the adjuvanted vaccine groups (rPvCS127: 2-6 and $\mathrm{rPvCS712:} \mathrm{8-12,}$ Table 1), we observed the highest levels of specific anti$\mathrm{rPvCS} 127$ and $-\mathrm{rPvCS} 712 \mathrm{IgG}$ antibodies in the groups 3 and 9 , respectively, which received antigen formulated 
(A) Anti-rPvCS127 and Anti-rPvCS712 Antibodies

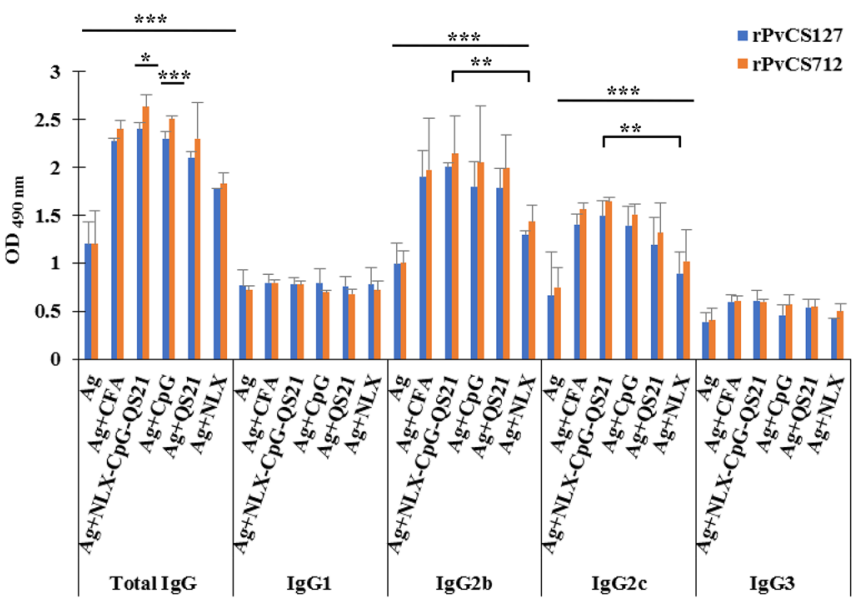

(B) Subclass Antibodies' Ratio

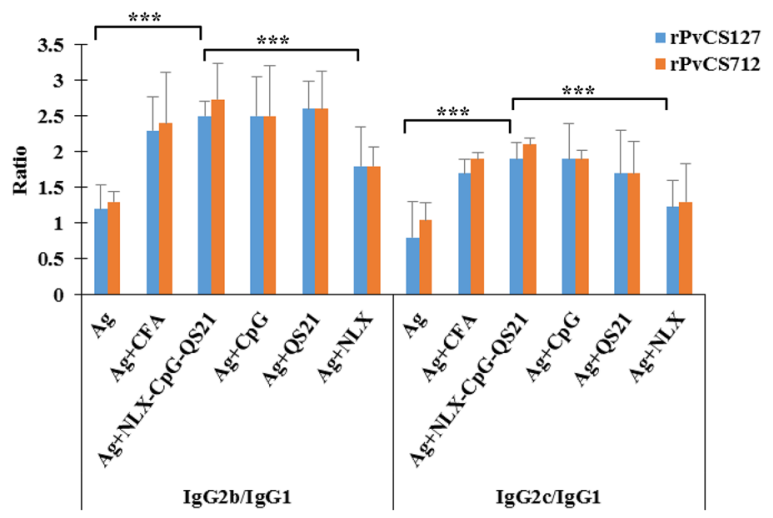

Fig. 2 Evaluation and comparison of the total $\mathrm{IgG}$ and IgG subclass antibodies to $\operatorname{rPvCS} 127$ and $\operatorname{rPvCS712}$ (a) in the sera of vaccinated mice by ELISA and (b) the ratio of $\mathrm{IgG} 2 \mathrm{~b} / \mathrm{IgG} 1$ and $\mathrm{IgG} 2 \mathrm{c} / \mathrm{IgG} 1$ anti-rPvCSP antibodies (Th1/ Th2 responses). Anti-rPvCS127 and -rPvCS712 total IgG and its subclasses were assessed in immunized mice on day 38 after the first immunization (10 days after the second boost). IgG2b and $\mathrm{IgG} 2 \mathrm{c}$ were the predominant isotypes in all the vaccine groups (1-12) and the highest levels of these prevalent subclasses were detected in the groups that received $\mathrm{PPvCS} 127$ or $\mathrm{PPvCS} 712$ with NLX-CpGQS21 combination $(P<0.0001$, one-way ANOVA). A significant difference in total $\mathrm{IgG}$ of immunized mouse groups was observed in the groups that received recombinant antigens $(\mathrm{rPvCS} 127$ or rPvCS712) formulated with $\mathrm{CpG}(P<0.0001$, Tukey's HSD post hoc test) and NLX-CpG-QS21 $(P<0.05$, Tukey's HSD post hoc

with NLX-CpG-QS21 combination $(P<0.0001$, one-way ANOVA; Fig. 2a). In addition, the multiple comparisons of serum-specific IgG levels in the vaccine groups (rPvCS127: 1-6 and rPvCS712: 7-12) showed a statistically significant increase in the level of anti-rPvCS712 $\mathrm{IgG}$ antibodies in the mouse groups receiving $\mathrm{rPvCS712}$ antigen either formulated with NLX-CpG-QS21 combination (group 9) or in the $\mathrm{CpG}$ adjuvant alone (group 10) $(P<0.0001$, Tukey's HSD post hoc test, Fig. 2a and Supplementary Table S1). Besides, specific anti-PvCSP IgG antibodies were observed in the vaccine groups 3 and 9 (receiving $\mathrm{rPvCS} 127$ or $\mathrm{PvCS} 712$ formulated with NLX-CpG-QS21 combination) and vaccine groups 2 and 8 (receiving antigens in CFA/IFA as a reference adjuvant) $(P>0.05$, Tukey's HSD post hoc test, Fig. 2a and Supplementary Table S1). Control mice that were immunized with only adjuvant(s) did not present the detectable specific antibodies (Fig. 1a, b). Furthermore, immunoblot results, using pooled sera derived from each immunized mouse group receiving either antigen alone or in combination with different adjuvants, showed that anti-rPvCS127 test). The ELISA cutoffs were calculated as the mean $\mathrm{OD}_{490 \mathrm{~nm}}$ of pre-immune mouse sera (as negative controls, $n=20$ ) plus 3SD. The cutoffs for anti-rPvCS127 IgG1, IgG2b, IgG2c, and IgG3 on day 38 were $\mathrm{OD}_{490 \mathrm{~nm}}=0.16,0.2,0.183$, and 0.184 , respectively. The cutoffs for anti-rPvCS712 IgG1, IgG2b, IgG2c, and IgG3 on day 38 were $\mathrm{OD}_{490 \mathrm{~nm}}=0.182,0.232,0.2$, and 0.222 , respectively. Among adjuvanted vaccine groups, rPvCS712+NLX-CpG-QS21 showed the highest $\operatorname{IgG} 2 \mathrm{~b} / \mathrm{IgG} 1$ and $\mathrm{IgG} 2 \mathrm{c} / \mathrm{IgG} 1$ ratio; however, the lowest $\mathrm{IgG} 2 \mathrm{~b} / \mathrm{IgG} 1$ and $\mathrm{IgG} 2 \mathrm{c} / \mathrm{IgG} 1$ ratio was observed in the groups $6(\mathrm{rPvCS} 127+\mathrm{NLX})$ and $12(\mathrm{rPvCS} 712+\mathrm{NLX})$, respectively $(P<0.0001$, one-way ANOVA). Data were analyzed using oneway ANOVA, followed by Tukey's HSD post hoc test. $* P<0.05$, $* * P \leq 0.001, * * * P<0.0001$. Ag: either $\mathrm{rPvCS} 127$ or $\mathrm{rPvCS} 712$ antigen

or $-\mathrm{rPvCS} 712 \mathrm{IgG}$ antibodies recognized the purified rPvCS127 or rPvCS712 antigen (Supplementary Fig. S2).

\section{IgG subclasses}

Analysis of distribution frequencies of specific IgG subtypes (anti-rPvCS127 or -rPvCS712 antibodies) in all the vaccine groups (1-12) on day 38 after the first immunization showed a bias toward $\operatorname{IgG} 2 b>\operatorname{IgG} 2 \mathrm{c}>\operatorname{IgG} 1>\operatorname{IgG} 3$ subclasses antibodies (Fig. 2a). Comparison between the non-adjuvanted ( 1 and 7) and adjuvanted (2-6 and 8-12) vaccine groups revealed a significant increase in the level of specific antiPvCSP IgG2 $b$ and $\operatorname{IgG} 2 \mathrm{c}$ antibodies in the adjuvanted vaccine groups $(P<0.0001$, one-way ANOVA; Fig. 2a). In addition, among the adjuvanted vaccine groups, a statistically significant difference was observed in the levels of IgG2b and $\mathrm{IgG} 2 \mathrm{c}$ antibodies between the groups that received each recombinant antigen with NLX-CpG-QS21 combination (3 and 9) and with NLX adjuvant alone (6 and 12) $(P<0.05$, Tukey's HSD post hoc test; Fig. 2a and Supplementary Table S1). Multiple comparisons of the vaccine groups with two antigens (rPvCS127: 1-6 and rPvCS712: 8-12) showed 
no significant difference in the levels of anti-rPvCS127 and -rPvCS712 IgG2b and IgG2c subclass antibodies $(P>0.05$, Tukey's HSD post hoc test; Fig. 2a and Supplementary Table S1). In addition, comparable specific anti-PvCSP $\mathrm{IgG} 2 \mathrm{~b}$ and $\mathrm{IgG} 2 \mathrm{c}$ antibodies were observed in the vaccine groups 3 and 9 that received $\mathrm{PPCS} 127$ or $\mathrm{rPvCS} 712$ in three human-use compatible vaccine adjuvants (NLX-CpG-QS21) and also the vaccine groups 2 and 8 that received antigens in CFA/IFA as a reference adjuvant $(P>0.05$, Tukey's HSD post hoc test, Fig. 2a and Supplementary Table S1).

Comparison of the ratio of Th1/Th2 antibodies among the non-adjuvanted ( 1 and 7$)$ and the adjuvanted (2-6 and $8-12$ ) vaccine groups showed the highest significant $\mathrm{IgG} 2 \mathrm{~b} /$ IgG1 (2.74) and $\mathrm{IgG} 2 \mathrm{c} / \mathrm{IgG} 1$ (2.1) ratios among the mouse group that received rPvCS712 formulated with NLX-CpGQS21 combination (group 9; Fig. 2b). The lowest IgG2b/ IgG1 (1.2 and 1.3) and IgG2c/IgG1 (0.8 and 1.04) ratios were observed in the non-adjuvanted vaccine groups 1 and 7, which received $\mathrm{rPvCS} 127$ or $\mathrm{rPvCS712}$ antigen alone, respectively (Fig. 2b).

\section{Antibody avidity of anti-rPvCS127 and -rPvCS712 IgG and its subclasses}

In this study, high-avidity $\operatorname{IgG}, \operatorname{IgG} 2 b$, and $\operatorname{IgG} 2 \mathrm{c}$ antibodies induced in the adjuvanted vaccine groups receiving each recombinant antigen formulated with NLX-CpG-QS21 combination (groups 3 and 9), $\mathrm{CpG}$ alone (groups 4 and 10), and QS21 alone (groups 5 and 11) on day 38 after the primary immunization (Fig. 3). By comparing all the vaccine mouse groups (rPvCS127: 1-6 and rPvCS712: 7-12), we found a statistically significant increase in the high-avidity $\operatorname{IgG}$ and IgG2c antibodies in the groups 9 and 10, which received rPvCS712 formulated with NLX-CpG-QS21 combination or CpG adjuvant alone, respectively $(P<0.05$, Tukey's HSD post hoc test; Fig. 3 and Supplementary Table S1). However, for IgG2b avidity, a significant increase in the high-avidity antibodies was observed in mice from group 10 that received rPvCS712 formulated with CpG adjuvant alone $(P<0.0001$, Tukey's HSD post hoc test; Fig. 3 and Supplementary Table S1). Concerning rPvCS127, the group receiving NLX adjuvant alone (group 6) showed a significant increase in the IgG and IgG2b avidities $(P<0.05$, Tukey's HSD post hoc test; Fig. 3 and Supplementary Table S1). Intermediate AI for $\mathrm{IgG}, \mathrm{IgG} 2 \mathrm{~b}$, and IgG2c was detected in the non-adjuvanted groups ( 1 and 7$)$ and the adjuvanted vaccine groups that formulated with NLX (6 and 12) on day 38 after the first immunization (Fig. 3). Besides, a statistically significant increase in the $\mathrm{IgG}$ and $\mathrm{IgG} 2 \mathrm{c}$, but not in $\mathrm{IgG} 2 \mathrm{~b}$ avidity, was found in vaccine groups 3 and 9 (received rPvCS127 or rPvCS712 in three human-use compatible vaccine adjuvants, NLX-CpG-QS21) and also vaccine groups 2 and 8

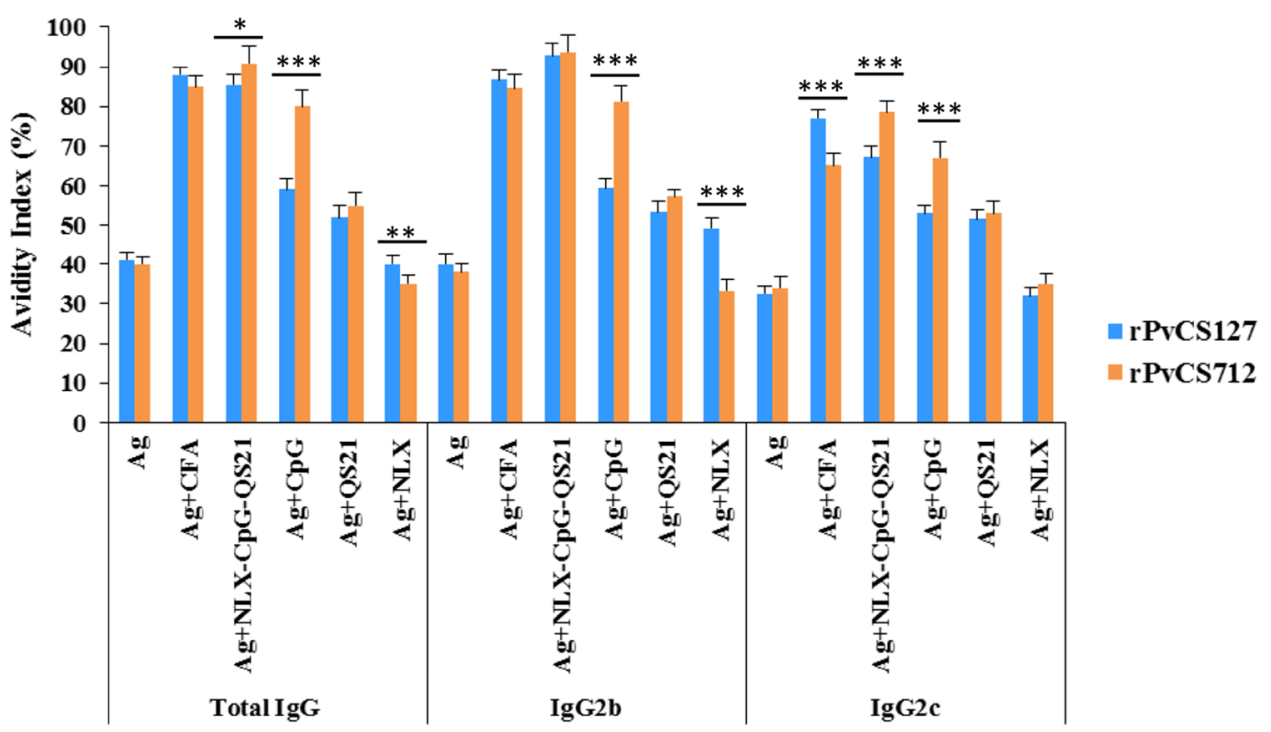

Fig. 3 Evaluation of anti-rPvCS127 and -rPvCS712 antibody avidity in immunized mice on day 38 after the first immunization by ELISA. High-avidity $\mathrm{IgG}, \mathrm{IgG} 2 \mathrm{~b}$, and $\mathrm{IgG} 2 \mathrm{c}$ antibodies were induced in vaccine groups except for the mouse groups that received $\mathrm{rPvCS} 127$ or rPvCS712 alone or in combination with NLX, as adjuvant. AI values $\leq 30 \%$, between 30 and $50 \%$, and $>50 \%$ were considered as low-, intermediate- and high-avidity anti-rPvCSP-specific antibodies, respectively. Multiple comparisons of the vaccine groups with two antigens (rPvCS127: 1-6 and rPvCS712: 8-12) showed a statistically significant difference in the IgG and IgG2c, but not IgG2b avidity in the vaccine groups 3 and 9 that received $\mathrm{rPvCS712}$ or $\mathrm{rPvCS712}$ in three human-use compatible vaccine adjuvants, NLX-CpG-QS21 $(P<0.05$, Tukey's HSD post hoc test). However, non-adjuvanted vaccine groups (1 and 7) showed no significant difference in the level of anti-rPvCS127 and -rPvCS712 IgG, IgG2b and IgG2c avidity antibodies $(P>0.05$, Tukey's HSD post hoc test). Data were analyzed using one-way ANOVA followed by Tukey's HSD post hoc test. $* P<0.05, * * P \leq 0.001, * * * P<0.0001$ 
(received antigens in CFA/IFA as a reference adjuvant) $(P<0.05$, Tukey's HSD post hoc test; Fig. 3 and Supplementary Table S1). However, concerning rPvCS127, a significant decrease was observed in IgG2c avidity only in group 3 (rPvCS127 + NLX-CpG-QS21) rather than the reference adjuvant CFA/IFA vaccine group $2(P<0.0001$, Tukey's HSD post hoc test; Fig. 3 and Supplementary Table S1).

\section{Cellular immune response analysis}

Both proliferation and cytokine production in the cultured splenocyte cells of all vaccine groups (1-12, Table 1$)$ were determined in vitro. On day 42 after the first immunization, three mice from each group were anesthetized, followed by cervical dislocation, and the splenocytes were used for further analysis. Both recombinant proteins were able to elicit lymphoproliferation of cultured splenocyte cells of the vaccine groups with different levels (Fig. 4).

Supernatants of the cultured spleen cells from the vaccine groups were analyzed for the presence of the cytokines IFN- $\gamma(120 \mathrm{~h})$, IL-10 (72 h), and IL-4 (24 and $48 \mathrm{~h})$ using murine cytokine immunoassay kits (R\&D System). The level of IFN- $\gamma$ in the supernatants of cultured spleen cells from the mice immunized with $\mathrm{rPvCS} 712$ alone was higher than the mouse group receiving rPvCS127 alone (Fig. 5a). Besides, comparing the level of IFN- $\gamma$ between the non-adjuvanted ( 1 and 7$)$ and the adjuvanted (2-6 and 8-12) vaccine groups

\section{Lymphocyte proliferation}

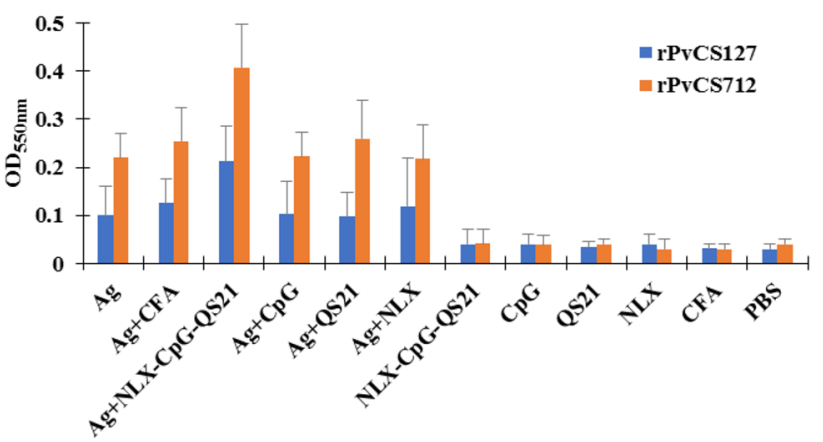

Fig. 4 Lymphocyte proliferation in cultured splenocyte cells of immunized mice (groups 1-12) in the presence of $\mathrm{rPvCS} 127$ and rPvCS712 in vitro. On day 42 after the first immunization, spleens from each vaccine (1-12) and control (13-18) groups (three mice per group) were removed, and lymphocyte proliferation was evaluated using the MTT assay. The bars show the mean OD values of lymphocytes proliferation of immunized mice $(n=3)$ in each group, and error bars indicate the SD of eight replicates. Lymphocyte proliferation in mice that received the antigens emulsified with different adjuvant formulas (2-6 and 8-12) was compared and both recombinant proteins were able to elicit lymphoproliferation of cultured splenocyte cells of the vaccine groups with different levels. Ag: either $\operatorname{rPvCS} 127$ or $\mathrm{rPvCS} 712$ antigen showed that the lowest level of IFN- $\gamma$ (means: 441 and $588 \mathrm{pg} / \mathrm{mL}$, respectively) was produced by the cultured spleen cells (at $120 \mathrm{~h}$ ) from the non-adjuvanted immunized mice (Fig. 5a). However, in the adjuvanted vaccine groups (rPvCS127: 2-6 and rPvCS712: 8-12), high levels of IFN- $\gamma$ were elicited in the groups immunized with $\mathrm{rPvCS} 127$ or rPvCS712 antigen formulated with NLX-CpG-QS21 combination (groups 3 and 9, mean: 1200 and $3092 \mathrm{pg} / \mathrm{mL}$, respectively; Fig. 5a). Also, higher levels of IFN- $\gamma$ were observed in the groups that received rPvCS712 (groups 8-12) rather than $\mathrm{PPCS} 127$ (groups 2-6) with different adjuvants (Fig. 5a). By comparing the adjuvanted vaccine groups (rPvCS127: 2-6 and rPvCS712: 8-12), we found that the group 9 (receiving rPvCS712 in three adjuvants, NLX-CpG-QS21) had the highest level of IFN- $\gamma$ (Fig. 5a). We also observed an increase in IFN- $\gamma$ production in vaccine groups 3 and 9 that received each recombinant antigen formulated in combination with three human-use compatible vaccine adjuvants (NLX-CpG-QS21) rather than vaccine groups 2 and 8 that received the reference adjuvant CFA/IFA (Fig. 5a). Control mouse groups (13-18) immunized with only adjuvant(s) did not present substantial IFN- $\gamma$ (Fig. 5a). Concerning the levels of IL-4 secretion, as the Th2 response cytokine, we found a lower level of IL-4 production in cultured spleen cells from different immunized mice (1-12) than the control groups (13-18), which were immunized with CpG, QS21, NLX, NLX-CpG-QS21, CFA/IFA, or PBS adjuvants (Supplementary Fig. S3) in the samples collected either at 24 or $48 \mathrm{~h}$. Regarding IL-10 secretion as T regulatory $\left(T_{\text {reg }}\right)$ response, the levels of IL-10 were $222 \mathrm{pg} / \mathrm{mL}$ and $85 \mathrm{pg} / \mathrm{mL}$, respectively, in the cultured spleen cells (at $72 \mathrm{~h}$ ) from immunized mice that received rPvCS127 (group 3) or rPvCS712 (group 9) in combination with three adjuvants, NLX-CpG-QS21 (groups 3 and 9; Fig. 5b).

\section{Discussion}

Although significant progression has been made in the development of vaccines against malaria, an effective malaria vaccine is not yet available, and progress in developing such a vaccine is moving slowly [64]. Unfortunately, to date, in phase I clinical trials, $P$. vivax CSP-based vaccines have been disappointing, especially when using recombinant proteins expressed in E. coli and Saccharomyces cerevisiae [65, 66] and using long synthetic peptides representing the immunogenic regions present in CSP $[67,68]$. This result is due to the poor immunogenicity of tested antigens that are not able to elicit inhibitory antibody responses [65-68]. Therefore, attempts to develop an effective $P$. vivax vaccine are still undergoing in different laboratories.

Allelic variation occurs in PvCSP antigen, and there is a possibility that vaccination based on CSP could select 
Fig. 5 Assessment of IFN- $\gamma(\mathbf{a})$ and IL-10 (b) in vaccine (1-12) and control (13-18) groups on day 42 after the first immunization. In $\mathbf{a}, \mathbf{b}$, the bars show the mean concentration of elicited IFN- $\gamma$ and IL- 10 from pooled lymphocytes of immunized mice $(n=3)$ in each group, and error bars indicate SD of eight replicates. For immunized mice receiving $\mathrm{rPvCS} 127$ with different adjuvants, the mean of IFN- $\gamma$ responses of ConA (as the positive control) and no antigen (as the negative control) was in the range of 920-3050 and $10-30 \mathrm{pg} / \mathrm{mL}$, respectively, but for rPvCS712 groups, they were 2160-3750 and 10-35 pg/ $\mathrm{mL}$, respectively. Different levels of IFN- $\gamma$ production were observed in the adjuvanted vaccine groups (2-6 and 8-12) on day 42 after the first immunization. The lowest level of IFN- $\gamma$ was produced by the non-adjuvanted vaccine groups. Among different examined groups, IL-10 responses of ConA (as the positive control) and no antigen (as the negative control) were in the range of 120-250 and $20-49 \mathrm{pg} / \mathrm{mL}$, respectively

\section{(A) IFN- $\gamma$}

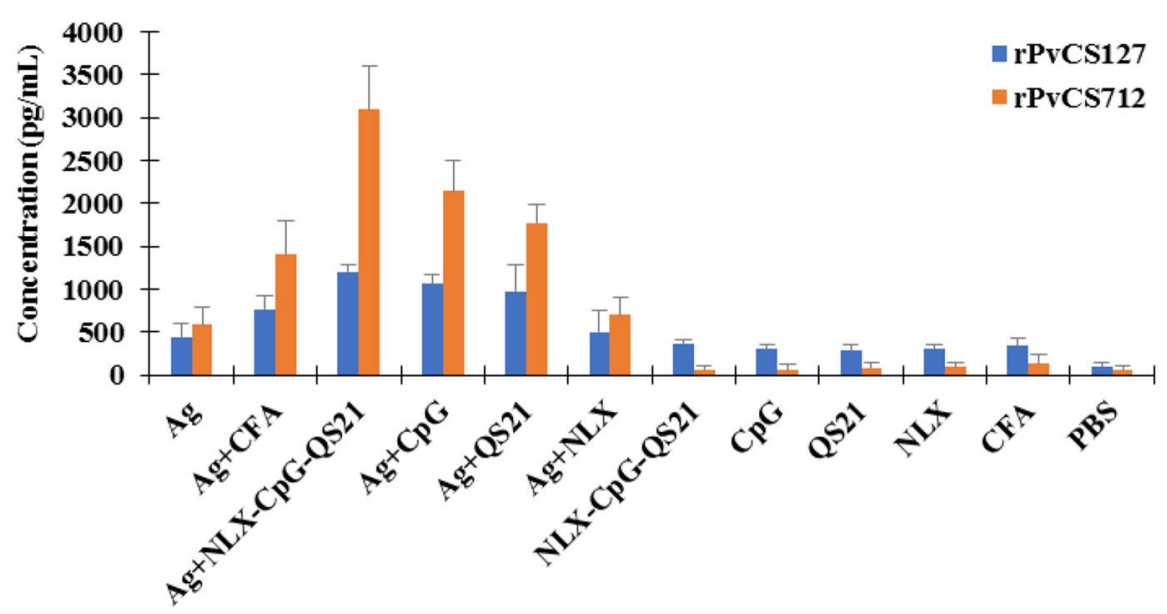

(B) IL-10

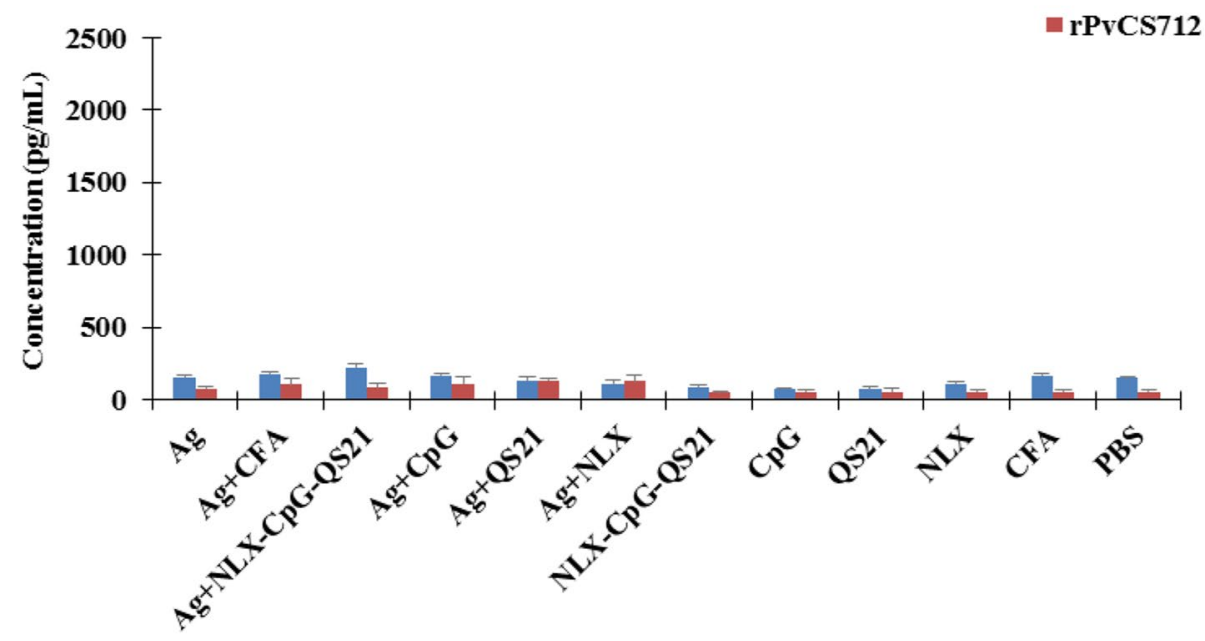

escape mutants. Therefore, a PvCSP-based vaccine should be able to target all the field isolates; in this regard, a multiallelic combination vaccine could address the problems of antigenic variation and help induce protective immunity against heterogeneous parasite populations and also handle the possible immune escape of the parasite. Therefore, in our previous work [62], we designed and expressed P. vivax chimeric recombinant proteins ( $\mathrm{rPvCS} 127$ and $\mathrm{rPvCS} 712)$ containing predicted $\mathrm{B}$ - and $\mathrm{T}$-cell epitopes derived from the $\mathrm{N}$ - and C-terminal regions of the native CSP protein and also the amino acid sequences representing the two major variant repeats of VK210 and VK247 types. Both designed constructs had a conformational structure that allowed recognition by two repeat type-specific mAbs and also by sera from individuals naturally infected by $P$. vivax. Moreover, anti-PvCS127 and -PvCS712 sera of immunized mice recognized the native form of the PvCSP antigen on sporozoite surface using IFAT [62]; this finding indicates the similarity of epitopes in recombinant forms to the corresponding native protein of both sporozoite types. These two chimeric proteins are bound to both heparin sulfate and HepG2 cells; hence, it can be concluded that both have the potential to be strong candidates for an anti-vivax vaccine [62].

In the continuation of our previous work, in the present investigation, to determine and compare whether such a chimeric protein expressed in E. coli has the ability to induce high-avidity titers of cytophilic subclass antibodies, we evaluated and compared the immunogenicity of both $\mathrm{rPvCS} 127$ 
and $\mathrm{rPvCS} 712$ in a murine model. The present results confirmed that both engineered proteins were immunogenic even though there was only a minor difference in epitopespecific antibody responses between the two constructs with modified epitope arrangement. This result also verifies that the conformation of target epitopes and/or interference with antigen processing and presentation are preserved in both engineered PvCS127 and PvCS712. Additionally, these two different arrangements of the repeat sequences of VK210 and VK247 in both $\mathrm{rPvCS} 127$ and $\mathrm{rPvCS} 712$ constructs, which contain B-cell epitopes, are accessible for the induction of adequate and proper immune responses.

Development of a new generation of potent subunit vaccines against intracellular pathogens relies on the choice of appropriate adjuvants to improve pathogen-specific humoral and cellular immunity [21]. It has been proven that the immunogenicity of the purified recombinant protein is low due to inactivation or loss of pathogens' essential immunostimulators (as a natural innate immune trigger) during purification processes as well as due to rapid degradation in vivo [54]. Therefore, to enhance the immunogenicity of purified recombinant antigens, there is a strong demand for the use of adjuvants [69]. In the present work, both candidate chimeric antigens ( $\mathrm{rPvCS} 127$ and $\mathrm{rPvCS} 712$ ) were immunogenic and elicited antigen-specific humoral and cellular responses in vivo. Immunized mice receiving either rPvCS127 or rPvCS712 alone (non-adjuvanted) induced almost an equal level of $\operatorname{IgG} 2 \mathrm{c}$ and $\mathrm{IgG} 1$, indicating mixed Th1/Th2 responses. However, immunization of mice with rPvCS127 and rPvCS712 formulated with either NLX-CpGQS21 combination or each adjuvant individually induced relatively high levels of $\operatorname{IgG} 2 b$ and $\operatorname{IgG} 2 \mathrm{c}$ as well as lower amounts of IgG1 and IgG3, indicating a Th1-polarized response. However, this finding was in contrast with previous reports [70,71] in which the Pichia pastoris-expressed chimeric proteins of PvCSP variants containing the three central repeat regions of different CSP alleles (VK210, VK247, and P. vivax-like) formulated with either Poly (I:C) or Montanide ISA720 adjuvant induced high levels of IgG1specific antibody (Th2 response) with intermediate avidity for all three $P$. vivax CSP variants in C57BL/6 mice. Therefore, the results of the present investigation indicate that although chimeric protein alone may reveal some degree of the immunogenicity, its combination with different Th1 potent adjuvants with distinct mechanisms could improve their immunogenicity in inducing Th1 response, which is more favorable for the pre-erythrocyte stage of a malaria vaccine.

The ability of antibodies to neutralize and clear malaria parasites depends on their specificity and subclass antibodies. Our previous work [62] revealed that in Iranian malaria-exposed subjects, the cytophilic anti-rPvCS127- and -rPvCS712-specific IgG1 and IgG3 were the predominant
IgG isotypes. This evidence confirms that modifications introduced in both chimeric proteins preserve the conformation of B-cell epitopes; therefore, both chimeric proteins are highly antigenic and can be recognized by sporozoiteinduced antibodies in natural infection. Interestingly, in the present work, we also found the murine equivalent antibodies $(\operatorname{IgG} 2 \mathrm{a} / \mathrm{c}$ and $\operatorname{IgG} 2 \mathrm{~b})$ to human IgG1 and IgG3 that possibly might have the ability to fix, complement and bind protein antigens. These murine results have also been supported by the high production of IFN- $\gamma$ in immunized mice that might not only modulate class switch recombination but also activate macrophages to enhance the phagocytosis of opsonized sporozoites [72, 73]. Besides, earlier works have reported that IFN- $\gamma$ produced by CS-specific CD4+ T-cells is a potent inhibitor of intracellular hepatic stages of Plasmodia [74, 75] and correlates with protection against $P$. falciparum sporozoite challenge in vaccinated $[27,76]$ and naturally infected individuals [77].

The immune sera from mouse vaccinated with each chimeric antigen, $\mathrm{rPvCS} 127$ or $\mathrm{rPvCS} 712$ plus different adjuvants had similar avidity IgG antibody, which is likely due to the maturation of the immune response and specific B-cell clonal selection that results in avidity increase [78]. In the mouse groups receiving both antigens alone (nonadjuvanted), anti-CSP IgG antibody with an intermediate avidity was detected; this result suggests that the adjuvants $\mathrm{CpG}$ and QS21 have a key role in the maturation of specific antibody responses against both chimeric antigens with high avidity and these features can be considered in the development of the rPvCSP-based vaccine. Regarding IgG2b and IgG2c (Th1 response), adjuvants CpG and QS21 induced higher avidity of anti-rPvCS127 or -rPvCS712 antibodies than NLX adjuvant in immunized mice. Thus, we can draw the conclusion that the strength of antibody-eliciting responses in mice against engineered $\mathrm{rPvCSP}$ could increase the avidity of the anti-rPvCSP antibodies when combined with either CpG or QS21 as an adjuvant. However, the role of such antibodies in blocking sporozoite invasion to hepatocyte needs further investigation.

The present results are consistent with the previous investigations suggesting that NLX [33, 34], CpG [79, 80], and QS21 [50, 52, 81] have the ability to induce Th1 immune response. In fact, $\mathrm{CpG}$ induces the expression of IL-12 by macrophages [82] and also indirectly activates NK cells and stimulates the IFN- $\gamma$ production [83-85]. The induction of IFN- $\gamma$ and IL-12 (which promote Th1 responses), but not IL-4 (which promotes Th2 responses) suggests that the administration of $\mathrm{CpG}$ in vivo might produce an environment favoring a Th1 immune response. Also, QS21 stimulated the induction of Th1 cytokines, IL- 2 , and IFN- $\gamma$, as well as the antibodies of the IgG2a isotype to the target protein antigens [50, 52, 55-57]. Besides, it has been proposed that IL-10 cytokine, as an immunoregulator of the infections 
caused by Plasmodium, has a significant role in deactivating the effects of other cytokines produced by Th1 cells [86-88].

Adjuvants can lead to the reduction of dose and/or number of boost for a given vaccine candidate $[89,90]$. In this regard, we found that after the second and the third immunizations (the first and the second boosts) of mice with both engineered antigens formulated in Th1 potent adjuvants, the magnitude and the quality of antigen-specific humoral and cellular responses were enhanced in a similar manner. These results may indicate the features of dose reduction in the antigen amount for vaccine formulation that could have important implications for improving global vaccine supply with a reasonable price for an estimated 3.3 billion people whom are at the risk of malaria [91] and need malaria vaccination.

In a previous work by Shabani et al. [62], it was reported that both designed constructs have structurally different characteristics at the molecular level, with a better expression level and higher recombinant protein yield in the case of rPvCS712, which suggests that this construct can provide high flexibility in terms of scaling-up and cost for large-scale vaccine production. Also, both chimeric proteins recognized by sera from the individuals naturally infected by $P$. vivax and anti-rPvCS127 and -rPvCS712 antibodies were able to recognize native protein on sporozoite surface, as shown by IFAT [62]. However, the highest IgG2b/IgG1 (2.74) and IgG2c/IgG1 (2.1) ratio was detected in the mouse groups immunized with rPvCS712 + NLX-CpG-QS21 rather than rPvCS127 + NLX-CpG-QS21. In addition, by comparing the mouse groups immunized with an equal amount of both chimeric antigens, it was found that the CSP-specific $\mathrm{CD} 4^{+}$ T-cells induced by rPvCS712+ NLX-CpG-QS21 (group 9) promoted the production of a higher level of IFN- $\gamma$ and a lower level of IL-10 compared to rPvCS127 + NLX-CpGQS21 (group 3). While there was an increase in pro-inflammatory Th1 cytokine (IFN- $\gamma$ ) levels in mice immunized with rPvCS712 + NLX-CpG-QS21, both chimeric antigens combined with NLX-CpG-QS21 as adjuvants were able to produce a considerable amount of pro-inflammatory Th1 cytokine, which may help the elimination of $P$. vivax. Although there is no explanation for this discrepancy in immune responses to these two chimeric proteins at the time of writing this manuscript, it could be postulated that such discrepancy is likely associated with differences in antigenic characteristics of these two antigens. Since different arrangements of repeat types of VK210 and VK247 were used, linear and new discontinuous B-cell epitopes were predicted for both structures [62]. Therefore, these epitopes might have different availability to the immune system and/ or the activation of distinct immune responses, resulting in the stimulation of different immune responses.

In summary, both chimeric proteins in C57BL/6 mice were efficient immunogens and were capable of inducing similar patterns of the potent humoral and cellular immune responses with different levels. Although Th1 potent adjuvants such as CpG, QS21, and NLX increased the antiPvCS127- and -PvCS712-specific cytophilic antibodies (IgG2b and $\mathrm{IgG} 2 \mathrm{c}$ ), the combination of these three Th1 potent adjuvants (by means of different mechanisms) with chimeric proteins (specially with $\mathrm{PPVCS712}$ ) showed a better impact on the magnitude and quality of humoral responses (specific antibody subclasses, titer, and high avidity). Taken together, the present findings indicate that $\mathrm{PvCS} 127$ and rPvCS712 meet the criteria to be potentially useful vaccine candidates against $P$. vivax malaria. However, further investigation is required to find out whether anti-PvCS127 or -PvCS712 antibodies are efficient in blocking P. vivax sporozoite invasion to hepatocyte.

Acknowledgements This work was financially supported by a Grant (no. 720) from Pasteur Institute of Iran to S. Zakeri and also partially supported by a Ph.D. scholarship to Samaneh H. Shabani from Zanjan University of Medical Sciences (A-12-190-11; Zanjan, Iran). We gratefully acknowledge François Nosten and Chiara Andolina at Mahidol Oxford Tropical Medicine Research Unit, Thailand for providing slides of $P$. vivax sporozoites. The authors also thank Mrs. M. Saffari for English editing of the manuscript.

\section{Compliance with ethical standards}

Conflict of interest The authors declare that they have no conflict of interest.

Ethical approval All animal handling was in accordance with the ethical standards of the Laboratory Animal Science Department, Pasteur Institute of Iran.

\section{References}

1. World Health Organization (WHO) (2014) World malaria report 2014. World Health Organization, Geneva

2. Arévalo-Herrera M, Chitnis C, Herrera S (2010) Current status of Plasmodium vivax vaccine. Hum Vaccine 6(1):124-132

3. The malERA Consultative Group on Vaccines (2011) A research agenda for malaria eradication: vaccines. PLoS Med $8(1): \mathrm{e} 1000398$

4. Genton B, D'Acremont V, Rare L, Baea K, Reeder JC, Alpers MP, Müller I (2008) Plasmodium vivax and mixed infections are associated with severe malaria in children: a prospective cohort study from Papua New Guinea. PLoS Med 5:e127

5. Kochar DK, Das A, Kochar SK, Saxena V, Sirohi P, Garg S, Kochar A, Khatri MP, Gupta V (2009) Severe Plasmodium vivax malaria: a report on serial cases from Bikaner in northwestern. India Am J Trop Med Hyg 80:194-198

6. Barber BE, William T, Grigg MJ, Menon J, Auburn S, Marfurt J, Anstey NM, Yeo TW (2013) A prospective comparative study of knowlesi, falciparum and vivax malaria in Sabah Malaysia: high proportion with severe disease from Plasmodium knowlesi and Plasmodium vivax but no mortality with early referral and artesunate therapy. Clin Infect Dis 56:383-397

7. Heppner G (2013) The malaria vaccine-status quo 2013. Travel Med Infect Dis 11(1):2-7 
8. Pancake SJ, Holt GD, Mellouk S, Hoffman SL (1992) Malaria sporozoites and circumsporozoite proteins bind specifically to sulphated glycoconjugates. J Cell Biol 117:1351-1357

9. Hollingdale MR (1990) Anti-sporozoite antibodies. Bull World Health Organ 68:47-51

10. Rosenberg R, Wirtz RA, Lanar DE, Sattabongkot J, Hall T, Waters AP, Prasittisuk C (1989) Circumsporozoite protein heterogeneity in the human malaria parasite Plasmodium vivax. Science 245(4921):973-976

11. Qari SH, Shi YP, Povoa MM, Alpers MP, Deloron P, Murphy GS, Harjosuwarno S, Lal AA (1993) Global occurrence of Plasmodium vivax-like human malaria parasite. J Infect Dis 168:1485-1489

12. Gopinath R, Wongsrichanalai C, Cordón-Rosales C, Mirabelli L, Kyle D, Kain KC (1994) Failure to detect a Plasmodium vivax-like malaria parasite in globally collected blood samples. J Infect Dis 170(6):1630-1633

13. Bilsborough J, Baumgart K, Bathurst I, Barr P, Good MF (1997) Fine epitope specificity of antibodies to region II of the Plasmodium vivax circumsporozoite protein correlates with ability to bind recombinant protein and sporozoites. Acta Trop 65:59-80

14. Coppi A, Pinzon-Ortiz C, Hutter C, Sinnis P (2005) The Plasmodium circumsporozoite protein is proteolytically processed during cell invasion. J Exp Med 201(1):27-33

15. Herrera S, Corradin G, Arévalo-Herrera M (2007) An update on the search for a Plasmodium vivax vaccine. Trends Parasitol 23(3):122-128

16. Franke ED, Lucas CM, San Roman E, Wirtz RA (1992) Prevalence of antibody to the variant repeat of the circumsporozoite protein of Plasmodium vivax in Peru. Am J Trop Med Hyg 46:708-710

17. Park JW, Son JI, Hur JP, Jong HS, Hwangbo Y, Lee SW, Kee MK, Shin YH, Yang BG (2000) An outbreak of vivax malaria in Republic of Korea in 1999. Korean J Infect Dis 32(4):335-339

18. Franke ED, Lucas CM, San Roman E (1991) Antibody response of humans to the circumsporozoite protein of Plasmodium vivax. Infect Immun 59(8):2836-2838

19. Mata E, Salvador A, Igartua M, Hernández RM, Pedraz JL (2013) Malaria vaccine adjuvants: latest update and challenges in preclinical and clinical research. Biomed Res Int 2013:282913

20. Arama C, Troye-Blomberg M (2014) The path of malaria vaccine development: challenges and perspectives. J Intern Med 275(5):456-466

21. Lee S, Nguyen MT (2015) Recent advances of vaccine adjuvants for infectious diseases. Immune Net 15(2):51-57

22. Thompson EA, Ols S, Miura K, Rausch K, Narum DL, Spångberg M, Juraska M, Wille-Reece U, Weiner A, Howard RF, Long CA, Duffy PE, Johnston L, O’Neil CP, Loré K (2018) TLR-adjuvanted nanoparticle vaccines differentially influence the quality and longevity of responses to malaria antigen Pfs25. JCI Insight 3(10): 120692

23. Carvalho LJ, Daniel-Ribeiro CT, Goto H (2002) Malaria vaccine: candidate antigens mechanisms constraints and prospects. Scand J Immunol 56:327-343

24. Doolan DL, Martinez-Alier N (2006) Immune response to preerythrocytic stages of malaria parasites. Curr Mol Med 6:169-185

25. John CC, Moormann AM, Pregibon DC, Sumba PO, McHugh MM, Narum DL, Lanar DE, Schluchter MD, Kazura JW (2005) Correlation of high levels of antibodies to multiple pre-erythrocytic Plasmodium falciparum antigens and protection from infection. Am J Trop Med Hyg 73:222-228

26. John CC, Tande AJ, Moormann AM, Sumba PO, Lanar DE, Min XM, Kazura JW (2008) Antibodies to pre-erythrocytic Plasmodium falciparum antigens and risk of clinical malaria in Kenyan children. J Infect Dis 197:519-526
27. Kester KE, Cummings JF, Ofori-Anyinam O, Ockenhouse CF, Krzych U, Moris P, Schwenk R, Nielsen RA, Debebe Z, Pinelis E, Juompan L, Williams J, Dowler M, Stewart VA, Wirtz RA, Dubois MC, Lievens M, Cohen J, Ballou WR, Heppner DG (2009) Randomized double-blind phase $2 \mathrm{a}$ trial of falciparum malaria vaccines RTSS/AS01B and RTSS/AS02A in malaria-naive adults: safety efficacy and immunologic associates of protection. J Infect Dis 200(3):337-346

28. Gupta RK, Relyveld EH, Lindblad EB, Bizzini B, Efraim SB, Gupta CK (1993) Adjuvants-A balance between toxicity and adjuvanticity. Vaccine 11:293-306

29. Vogel FR (2000) Improving vaccine performance with adjuvants. Clin Infect Dis 30(3):S266-S270

30. Pashine A, Valiante NM, Ulmer JB (2005) Targeting the innate immune response with improved vaccine adjuvants. Nat Med 11(4):S63-S68

31. Perez-Mazliah D, Langhorne J (2015) CD4 T-cell subsets in malaria: TH1/TH2 revisited. Front Immunol 5:671

32. Sacerdote P, di San Secondo VE, Sirchia G, Manfredi B, Panerai AE (1998) Endogenous opioids modulate allograft rejection time in mice: possible relation with Th1/Th2 cytokines. Clin Exp Immunol 113:465-469

33. Sacerdote P, Gaspani L, Panerai AE (2000) The opioid antagonist naloxone induces a shift from type 2 to type 1 cytokine pattern in normal and skin-grafted mice. Ann N Y Acad Sci 917:755-763

34. Sacerdote P, Manfredi B, Gaspani L, Panerai AE (2000) The opioid antagonist naloxone induces a shift from type 2 to type 1 cytokine pattern in BALB/cJ mice. Blood 95(6):2031-2036

35. Jamali A, Mahdavi M, Shahabi S, Hassan ZM, Sabahi F, Javan M, Farsani MJ, Parsania M, Bamdad T (2007) Naloxone an opioid receptor antagonist enhances induction of protective immunity against HSV-1 infection in BALB/c mice. Microb Pathog 43(5-6):217-223

36. Jamali A, Mahdavi M, Hassan ZM, Sabahi F, Farsani MJ, Bamdad T, Soleimanjahi H, Motazakker M, Shahabi S (2009) A novel adjuvant the general opioid antagonist naloxone elicits a robust cellular immune response for a DNA vaccine. Int Immunol 21:217-225

37. Jazani NH, Karimzad M, Mazloomi E, Sohrabpour M, Hassan ZM, Ghasemnejad H, Roshan-Milani S, Shahabi S (2010) Evaluation of the adjuvant activity of aloxone an opioid receptor antagonist in combination with heat-killed Listeria mono-cytogenes vaccine. Microbes Infect 12:382-388

38. Motaharinia Y, Rezaee MA, Rashidi A, Jalili A, Rezaie MJ, Shapouri R, Hossieni W, Rahmani MR (2013) Induction of protective immunity against brucellosis in mice by vaccination with a combination of naloxone alum and heat-killed Brucella melitensis. M J Microbiol Immunol Infect 46:253-258

39. McCarthy L, Wetzel M, Sliker JK, Eisenstein TK, Rogers TJ (2001) Opioids opioid receptors and the immune response. Drug Alcohol Depend 62:111-123

40. Krieg AM (2002) CpG motifs in bacterial DNA and their immune effects. Annu Rev Immunol 20:709-760

41. Krieg AM, Yi AK, Matson S, Waldschmidt TJ, Bishop GA, Teasdale R, Koretzky GA, Klinman DM (1995) CpG motifs in bacterial DNA trigger direct B-cell activation. Nature 374:546-549

42. Klinman DM, Yi AK, Beaucage SL, Conover J, Krieg AM (1996) CpG motifs present in bacterial DNA rapidly induce lymphocytes to secrete interleukin 6 interleukin 12 and interferon \& \#x03B3. Proc Natl Acad Sci USA 93:2879-2883

43. Klinman DM, Ishii KJ, Verthelyi D (2000) CpG DNA augments the immunogenicity of plasmid DNA vaccines. Curr Top Microbiol Immunol 247:131-142

44. Krieg AM (2006) Therapeutic potential of Toll-like receptor 9 activation. Nat Rev Drug Discov 5:471-484 
45. Harandi AM, Holmgren J (2004) CpG DNA as a potent inducer of mucosal immunity: implications for immune prophylaxis and immunotherapy of mucosal infections. Curr Opin Investig Drugs 5(2):141-145

46. Zhu D, Tuo W (2016) QS-21: a potent vaccine adjuvant. Nat Prod Chem Res 3(4):e113

47. Fernández-Tejada A, Chea EK, George C, Pillarsetty N, Gardner JR, Livingston PO, Ragupathi G, Lewis JS, Tan DS, Gin DY (2014) Development of a minimal saponin vaccine adjuvant based on QS-21. Nat Chem 6(7):635-643

48. Kensil CR, Patel U, Lennick M, Marciani D (1991) Separation and characterization of saponins with adjuvant activity from Quillaja saponaria Molina cortex. J Immunol 146:431-437

49. Kensil CR (1996) Saponins as vaccine adjuvants. Crit Rev Ther Drug Carr Syst 13:1-55

50. Singh M, O'Hagan DT (2003) Recent advances in veterinary vaccine djuvants. Int J Parasitol 33:469-478

51. Garçon N, Chomez P, Van Mechelen M (2007) GlaxoSmithKline adjuvant systems in vaccines: concepts achievements and perspectives. Expert Rev Vaccines 6:723-739

52. Sun HX, Xie Y, Ye YP (2009) Advances in saponin-based adjuvants. Vaccine 27:1787-1796

53. Song X, Hu S (2009) Adjuvant activities of saponins from traditional Chinese medicinal herbs. Vaccine 27:4883-4890

54. Garçon N, Van Mechelen M (2011) Recent clinical experience with vaccines using MPL- and QS-21-containing adjuvant systems. Expert Rev Vaccines 10:471-486

55. Kensil CR, Kammer R (1998) QS-21: a water-soluble triterpene glycoside adjuvant. Expert Opin Investig Drugs 7:1475-1482

56. O'Hagan DT, MacKichan ML, Singh M (2001) Recent developments in adjuvants for vaccines against infectious diseases. Biomol Eng 18:69-85

57. Liu G, Anderson C, Scaltreto H, Barbon J, Kensil CR (2002) QS-21 structure/function studies: effect of acylation on adjuvant activity. Vaccine 20:2808-2815

58. Glauert AM, Dingle JT, Lucy JA (1962) Action of saponin on biological cell membranes. Nature 196(4858):953-955

59. Yadava A, Sattabongkot J, Washington MA, Ware LA, Majam V, Zheng H, Kumar S, Ockenhouse CF (2007) A novel chimeric Plasmodium vivax circumsporozoite protein induces biologically functional antibodies that recognize both VK210 and VK247 sporozoites. Infect Immun 75(3):1177-1185

60. Vanloubbeeck Y, Pichyangkul S, Bayat B, Yongvanitchit K, Bennett JW, Sattabongkot J, Schaecher K, Ockenhouse CF, Cohen J, Yadava A (2013) Comparison of the immune responses induced by soluble and particulate Plasmodium vivax circumsporozoite vaccine candidates formulated in AS01 in rhesus macaques. Vaccine 31:6216-6224

61. Bennett JW, Yadava A, Tosh D, Sattabongkot J, Komisar J, Ware LA, McCarthy WF, Cowden JJ, Regules J, Spring MD, Paolino K, Hartzell JD, Cummings JF, Richie TL, Lumsden J, Kamau E, Murphy J, Lee C, Parekh F, Birkett A, Cohen J, Ballou WR, Polhemus ME, Vanloubbeeck YF, Vekemans J, Ockenhouse CF (2016) Phase 1/2a trial of Plasmodium vivax malaria vaccine candidate VMP001/AS01B in malaria-Naive adults: safety, immunogenicity, and efficacy. PLoS Negl Trop Dis 10(2): $\mathrm{e} 0004423$

62. Shabani SH, Zakeri S, Salmanian AH, Amani J, Mehrizi AA, Snounou G, Nosten F, Andolina C, Mourtazavi Y, Djadid ND (2017) Biological immunological and functional properties of two novel multi-variant chimeric recombinant proteins of CSP antigens for vaccine development against Plasmodium vivax infection. Mol Immunol 90:158-171

63. Hedman K, Lappalainen M, Seppaia I, Makela O (1989) Recent primary toxoplasma infection indicated by a low avidity of specific IgG. J Infect Dis 159(4):736-740
64. Dhanawat M, Das N, Nagarwal RC, Pandit JK (2010) Development in malarial vaccine: a review. Drug Discov Ther 4(5):298-313

65. Gordon DM, Cosgriff TM, Schneider I, Wasserman GF, Majarian WR, Hollingdale MR, Chulay JD (1990) Safety and immunogenicity of a Plasmodium vivax sporozoite vaccine. Am J Trop Med Hyg 42:527-531

66. Herrington DA, Nardin EH, Losonsky G, Bathurst IC, Barr PJ, Hollingdale MR, Edelman R, Levine MM (1991) Safety and immunogenicity of a recombinant sporozoite malaria vaccine against Plasmodium vivax. Am J Trop Med Hyg 45:695-701

67. Herrera S, Bonelo A, Perlaza BL, Fernandez OL, Victoria L, Lenis AM, Soto L, Hurtado H, Acuna LM, Velez JD, Palacios R, Chen-Mok M, Corradin G, Arevalo-Herrera M (2005) Safety and elicitation of humoral and cellular responses in Colombian malaria-naive volunteers by a Plasmodium vivax circumsporozoite protein-derived synthetic vaccine. Am J Trop Med Hyg 73:3-9

68. Herrera S, Fernandez OL, Vera O, Cardenas W, Ramirez O, Palacios R, Chen-Mok M, Corradin G, Arevalo-Herrera M (2011) Phase I safety and immunogenicity trial of Plasmodium vivax CS derived long synthetic peptides adjuvanted with montanide ISA 720 or montanide ISA 51. Am J Trop Med Hyg 84:12-20

69. Girard MP, Reed ZH, Friede M, Kieny MP (2007) A review of human vaccine research and development: malaria. Vaccine 25(9):1567-1580

70. Gimenez AM, Lima LC, Françoso KS, Denapoli PMA, Panatieri R, Bargieri DY, Thiberge JM, Andolina C, Nosten F, Renia L, Nussenzweig RS, Nussenzweig V, Amino R, Rodrigues MM, Soares IS (2017) Vaccine containing the three allelic variants of the Plasmodium vivax circumsporozoite antigen induces protection in mice after challenge with a transgenic rodent malaria parasite. Front Immunol 11(8):1275

71. de Camargo TM, de Freitas EO, Gimenez AM, Lima LC, de Almeida Caramico K, Françoso KS, Bruna-Romero O, Andolina C, Nosten F, Rénia L, Ertl HCJ, Nussenzweig RS, Nussenzweig V, Rodrigues MM, Reyes-Sandoval A, Soares IS (2018) Primeboost vaccination with recombinant protein and adenovirus-vector expressing Plasmodium vivax circumsporozoite protein (CSP) partially protects mice against $\mathrm{Pb} / \mathrm{Pv}$ sporozoite challenge. Sci Rep 8(1):1118

72. Danforth HD, Aikawa M, Cochrane AH, Nussenzweig RS (1980) Sporozoites of mammalian malaria: attachment to interiorization and fate within macrophages. J Protozool 27(2):193-202

73. Schwenk R, Asher LV, Chalom I, Lanar D, Sun P, White K, Keil D, Kester KE, Stoute J, Heppner DG, Krzych U (2003) Opsonization by antigen-specific antibodies as a mechanism of protective immunity induced by Plasmodium falciparum circumsporozoite protein-based vaccine. Parasite Immunol 25(1):17-25

74. Ferreira A, Schofield L, Enea V, Schellekens H, van der Meide P, Collins WE, Nussenzweig RS, Nussenzweig V (1986) Inhibition of development of exoerythrocytic forms of malaria parasites by gamma-interferon. Science 232(4752):881-884

75. Nardin EH, Nussenzweig RS (1993) T cell responses to preerythrocytic stages of malaria: role in protection and vaccine development against pre-erythrocytic stages. Annu Rev Immunol 11:687-727

76. Sun P, Schwenk R, White K, Stoute JA, Cohen J, Ballou WR, Voss G, Kester KE, Heppner DG, Krzych U (2003) Protective immunity induced with malaria vaccine RTS, $\mathrm{S}$ is linked to Plasmodium falciparum circumsporozoite protein-specific CD4 + and CD8 + T cells producing IFN-gamma. J Immunol 171(12):6961-6967

77. Reece WH, Pinder M, Gothard PK, Milligan P, Bojang K, Doherty T, Plebanski M, Akinwunmi P, Everaere S, Watkins KR, Voss G, Tornieporth N, Alloueche A, Greenwood BM, Kester KE, McAdam KP, Cohen J, Hill AV (2004) A CD4(+) T-cell immune response to a conserved epitope in the circumsporozoite protein 
correlates with protection from natural Plasmodium falciparum infection and disease. Nat Med 10(4):406-410

78. Thomas HI, Wilson S, O'Toole CM, Lister CM, Saeed AM, Watkins P, Morgan-Capner P (1996) Differential maturation of avidity of $\mathrm{IgG}$ antibodies to gp41 p24 and p17 following infection with HIV-1. Clin Exp Immunol 103(2):185-191

79. Sugai T, Mori M, Nakazawa M, Ichino M, Naruto T, Kobayashi N, Kobayashi Y, Minami M, Yokota S (2005) A CpG-containing oligodeoxynucleotide as an efficient adjuvant counter balancing the Th1/Th2 immune response in diphtheria-tetanus-pertussis vaccine. Vaccine 23(46-47):5450-5456

80. Fogg CN, Americo JL, Lustig S, Huggins JW, Smith SK, Damon I, Resch W, Earl PL, Klinman DM, Moss B (2007) Adjuvantenhanced antibody responses to recombinant proteins correlates with protection of mice and monkeys to orthopoxvirus challenges. Vaccine 25(15):2787-2799

81. Moore A, McCarthy L, Mills KH (1999) The adjuvant combination monophosphoryl lipid A and QS21 switches T cell responses induced with a soluble recombinant HIV protein from Th2 to Th1. Vaccine 17(20-21):2517-2527

82. Chace JH, Hooker NA, Midlenstein KL, Krieg AM, Cowdery JS (1997) Bacterial DNA-induced NK cell IFN-g production is dependent on macrophage secretion of IL-12. Clin Immunol Immunopathol 84(2):185-193

83. Yamamoto S, Yamamoto T, Shimada S, Kuramoto E, Yano O, Kataoka T, Tokunaga T (1992) DNA from bacteria but not from vertebrates induces interferons activates natural killer cells and inhibits tumor growth. Microbiol Immunol 36(9):983-997

84. Ballas ZK, Rasmussen WL, Krieg AM (1996) Induction of NK activity in murine and human cells by $\mathrm{CpG}$ motifs in oligodeoxynucleotides and bacterial DNA. J Immunol 157(5):1840-1845
85. Cowdery JS, Chace JH, Yi AK, Krieg AM (1996) Bacterial DNA induces NK cells to produce IFN-g in vivo and increases the toxicity of lipopolysaccharides. J Immunol 156(12):4570-4575

86. Langhorne J, Ndungu FM, Sponaas AM, Marsh K (2008) Immunity to malaria: more questions than answers. Nat Immunol 9:725-732

87. Couper KN, Blount DG, Riley EM (2008) IL-10: the master regulator of immunity to infection. J Immunol 180:5771-5777

88. Hansen DS, Schofield L (2010) Natural regulatory T cells in malaria: host or parasite allies? PLoS Pathog 6:e1000771

89. Galli G, Medini D, Borgogni E, Zedda L, Bardelli M, Malzone C, Nuti S, Tavarini S, Sammicheli C, Hilbert AK, Brauer V, Banzhoff A, Rappuoli R, Del Giudice G, Castellino F (2009) Adjuvanted $\mathrm{H} 5 \mathrm{~N} 1$ vaccine induces early $\mathrm{CD} 4+\mathrm{T}$ cell response that predicts long-term persistence of protective antibody levels. Proc Natl Acad Sci USA 106(10):3877-3882

90. Banzhoff A, Gasparini R, Laghi-Pasini F, Staniscia T, Durando P, Montomoli E, Capecchi PL, di Giovanni P, Sticchi L, Gentile C, Hilbert A, Brauer V, Tilman S, Podda A (2009) MF59-adjuvanted $\mathrm{H} 5 \mathrm{~N} 1$ vaccine induces immunologic memory and heterotypic antibody responses in non-elderly and elderly adults. PLoS One 4(2):e4384

91. World Health Organization (WHO) (2015) World malaria report 2015. World Health Organization, Geneva

Publisher's Note Springer Nature remains neutral with regard to jurisdictional claims in published maps and institutional affiliations. 